\title{
Arterial stiffness and cardiometabolic health (Q) in omnivores and vegetarians: a cross-sectional pilot study
}

Selicia T. Mayra ${ }^{1 *}$ and Carol S. Johnston ${ }^{2}$

\begin{abstract}
Objective: Arterial stiffness is a strong predictor of cardiovascular mortality, and often precedes elevations in blood pressure. This cross-sectional pilot study examined differences in arterial stiffness, blood pressure, cardiometabolic markers, anthropometric outcomes, and inflammation in vegetarians and matched omnivores. Participants were healthy, non-smoking adults (18-65 years old) adhering to either a vegetarian/vegan or omnivore diet. Omnivores were matched to vegetarians using broad body mass index (BMI) categories.

Results: Arterial stiffness trended higher in omnivores versus vegetarians ( $7.0 \pm 1.5$ and $6.8 \pm 1.1 \mathrm{~m} / \mathrm{s}$, respectively; $p=0.073)$. This trend was mainly driven by the male omnivores $(p=0.006$ for gender effect and $p=0.294$ for eating pattern effect). Omnivores displayed higher HDL concentrations compared to vegetarians, $63.8 \pm 18.5$ and $55.2 \pm 16.9 \mathrm{mg} / \mathrm{dL}$; however, total cholesterol/HDL ratio did not vary significantly between groups; $\mathrm{p}=0.310$. In men, a vegetarian eating pattern may reduce arterial stiffness; however, this benefit may be limited in women, particularly those who are premenopausal. Future research should examine arterial stiffness and cardiometabolic health outcomes in younger versus older female vegetarians, as these data can provide valuable insights on the role of plantbased eating patterns on arterial stiffness and cardiometabolic health.
\end{abstract}

Keywords: Arterial stiffness, Vegetarian eating patterns, Aging, Plant-based diets, Nutrition, Cardiometabolic health

\section{Introduction}

Conditions affecting the cardiovascular system are the leading cause of mortality worldwide [1]. In 2019 alone, 17.9 million deaths were attributed to cardiovascular diseases (CVD), representing a global death toll of $32 \%$. Early detection of CVD risk is crucial for disease detection and amelioration. Arterial stiffening (e.g., the loss of elastic compliance in the vascular wall) is a strong predictor of cardiovascular mortality, and often precedes elevations in blood pressure. Aging is closely associated with arterial stiffness, and further evidence suggests

\footnotetext{
*Correspondence: stmayra@bsu.edu

${ }^{1}$ Department of Nutrition and Health Science, Ball State University, 1613

W Riverside Ave, Muncie, IN 47303, USA

Full list of author information is available at the end of the article
}

that unhealthful lifestyle practices, including high meat, sodium, and alcohol consumption, smoking, and sedentary behaviors also contribute to the progression of arterial stiffness [2-4].

Pulse wave velocity (PWV) represents a non-invasive marker of disease risk, and, to date, carotid-femoral pulse wave velocity (Cf-PWV) is the most studied technique to quantify arterial stiffness in population-based studies [5]. Cf-PWV is the velocity of the arterial pulse as it travels along the vessel wall and is measured from the carotid to the femoral artery [6]. Reference values range from 4.6 to $7.5 \mathrm{~m} / \mathrm{s}$ for young, healthy adults and increase progressively with age $(+200 \%$ between the second and ninth decade of life) [7, 8]. PWV values $>10 \mathrm{~m} / \mathrm{s}$ are considered high risk for cardiovascular events. 
This cross-sectional study examined associations between arterial stiffness (Cf-PWV), brachial artery and central aortic blood pressures, cardiometabolic markers, and inflammation by eating pattern type (vegetarian versus omnivore). We hypothesized that these biomarkers would be more favorably aligned to a vegetarian eating pattern compared to an omnivore diet.

\section{Methods \\ Study design}

Data collection for this cross-sectional study occurred from February to July 2019 and consisted of a single in-person visit to the Healthy Lifestyle research facility located in downtown Phoenix, Arizona, USA. Study outcomes were arterial stiffness (Cf-PWV); blood pressure [systolic blood pressure (SBP), diastolic blood pressure (DBP), central systolic blood pressure (CSBP), and central diastolic blood pressure (CDBP)]; cardiometabolic markers [total cholesterol, HDL and LDL cholesterol, and triglycerides], and inflammation [highsensitivity C-reactive protein (hs-CRP)]. Anthropometric measures [body weight, body mass index (BMI), and waist and hip circumferences], and physical activity [Godin-Shephard Leisure-Time Physical Activity Questionnaire] [9] were also recorded. To increase the validity of inferences and to reduce bias, the research team, including the research nurse and phlebotomist were blinded to group allocation, and data were analyzed by the principal investigator.

\section{Participants}

Healthy, non-smoking adults (18-65 years old) adhering to either a vegetarian/vegan or omnivore diet were recruited from a campus population. A vegetarian/ vegan self-identified as 'eating no meat, poultry, or fish' and were distinguished from individuals who reported consuming 'meat, fish, and/or poultry on occasion but not daily'. Pregnant or recently pregnant (within the past three months) women, lactating women, individuals with chronic health conditions as well as those taking medication for these conditions, and individuals engaged in $>150$ min per week of moderate to vigorous intensity physical activity were excluded from the study. To create comparable groups, omnivores were matched to vegetarians using broad BMI $(18.5$ to $<25.0$, 25.0 to $<30.0$, and $\left.\geq 30.0 \mathrm{~kg} / \mathrm{m}^{2}\right)$ and age $(18-25,30-40$, and $50-65$ years) categories. Written informed consent was obtained for all participants, and the study was approved by the Institutional Review Board at Arizona State University.

\section{Study outcomes}

\section{Arterial stiffness and blood pressure}

Participants were instructed to lay supine in a dimly lit, temperature-controlled room for $10 \mathrm{~min}$. A non-invasive SphygmoCor XCEL (AtCor Medical, Sydney, NSW, Australia) system assessed Cf-PWV. A blood pressure cuff was placed around each participant's upper thigh and brachial artery of the non-dominant arm. Carotid and femoral pulse rates were identified and recorded, and measured distances from the femoral pulse to the topmost point above of the blood pressure cuff, from the sternal notch to the topmost point above the blood pressure cuff, and from the carotid pulse to the sternal notch were entered into the SphygmoCor XCEL software. A tonometer was placed directly above the carotid pulse, and firm yet stable pressure was applied allowing the SphygmoCor XCEL device to compute CfPWV. Brachial artery and central aortic blood pressures were also recorded. Measurements were obtained in triplicate, and the mean of the last two measurements was recorded.

\section{Cardiometabolic markers and inflammation}

One $10 \mathrm{~mL}$ fasting (except water for $12 \mathrm{~h}$ ) blood sample was acquired from each participant's antecubital vein. Total cholesterol was measured using a cholesterol fluorometric assay kit (Cat. No. 10007640, Cayman Company, Ann Arbor, MI, USA), HDL cholesterol was assessed with a quantitation kit (Cat. No. MAK045-1KT, Millipore-Sigma, Burlington, MA, USA), LDL cholesterol was calculated using the Friedewald formula [10], triglycerides were measured using a Colorimetric Assay Kit (Cat. No. 10010303, Cayman Company, Ann Arbor, MI), and an ELISA kit (Cat. No.10011236) assessed hs-CRP.

\section{Anthropometry measurements}

Height $(\mathrm{cm})$ was recorded using a stadiometer (SECA 217, Tiger Medical, Irvington, NJ, USA). Weight (kg) was obtained using a research-grade, calibrated total body composition analyzer (Cat. No. TBF-300, Tanita, Arlington Heights, IL, USA). Waist and hip circumferences were obtained with a SECA ergonomic measuring tape (Cat. No. 2011717009, Tiger Medical, Irvington, NJ, USA). For waist circumference, the measuring tape was positioned horizontally around the abdomen at the midpoint between the lowest rib and the top of the iliac crest. For hip circumference, the measuring tape was positioned around the widest portion of the posterior, parallel to the floor. Measurements were obtained twice and averaged. 


\section{Statistical analysis}

Power analysis indicated that a sample size of 58 was suitable to detect a $0.6 \mathrm{~m} / \mathrm{s}$ difference in PWV at a $5 \%$ level of significance assuming a standard deviation of 0.8 [11]. Data are represented as mean \pm SD. Data that violated the Gaussian distribution were transformed prior to analyses (Cf-PWV, HDL cholesterol, hs-CRP, total cholesterol/HDL ratio, and triglyceride/HDL ratio). Group differences for Cf-PWF, brachial artery and central aortic blood pressures, and cardiometabolic markers were analyzed using univariate analyses controlling for age, adiposity, and gender. Additionally, a multivariate ANOVA was conducted for the cardiometabolic factors that were correlated (SBP, DBP, LDL cholesterol, total cholesterol, and triglycerides), and a two-way ANOVA was utilized to assess Cf-PWV by diet plan and gender. Descriptive data were compared using the Mann-Whitney $U$ test or the Chi-Square test. Spearman coefficients were computed to compare relationships between variables. All analyses were performed using the Statistical Package for the Social Sciences, version 27 (IBM Corp., Armonk, NY, USA), and statistical significance was established at a $\mathrm{p}$-value $\leq 0.05$

\section{Results}

\section{Participants}

Of 149 eligible respondents, 55 individuals agreed to enroll in the study and completed the in-person visit (22 omnivores and 33 vegetarians). Thirteen vegetarians self-reported occasional meat consumption and were excluded from analyses. The median duration of adherence to a vegetarian eating pattern was nine years (range: 2-42 years). Participants were 75\% Caucasian. Participant characteristics are displayed in Table 1; adiposity measures did differ by eating pattern. Gender, age, and BMI were significantly related to Cf-PWV, and these variables were controlled in the outcome analyses.

Table 1 Participant characteristics by eating pattern type*

\begin{tabular}{llll}
\hline & Omnivores & Vegetarians & p value \\
\hline Gender (M/F) & $5 / 17$ & $4 / 16$ & 0.830 \\
Age $(\mathrm{y})$ & $37.4 \pm 15.4$ & $34.2 \pm 12.5$ & 0.623 \\
Body weight $(\mathrm{kg})$ & $69.1 \pm 15.2$ & $64.2 \pm 9.4$ & 0.358 \\
BMl $\left(\mathrm{kg} / \mathrm{m}^{2}\right)$ & $24.4 \pm 3.9$ & $22.3 \pm 2.8$ & 0.030 \\
Waist circumference $(\mathrm{cm})$ & $76.2 \pm 11.7$ & $72.2 \pm 8.4$ & 0.242 \\
Hip circumference $(\mathrm{cm})$ & $100.8 \pm 8.2$ & $95.8 \pm 6.1$ & 0.030 \\
Physical activity score & $46.2 \pm 22.5$ & $58.2 \pm 54.3$ & 0.920 \\
\hline
\end{tabular}

${ }^{*}$ Data are mean $\pm S D$; $p$-values represent Mann-Whitney U test or Chi-Square test for gender

\section{Comparison of study outcomes by eating pattern type}

Cf-PWV trended higher in the omnivores compared to the vegetarians $(7.0 \pm 1.5$ and $6.8 \pm 1.1 \mathrm{~m} / \mathrm{s}$, respectively; $\mathrm{p}=0.073$ ) (shown in Table 2). Although, when separated by gender, the trend by eating pattern was mainly driven by the male participants; $\mathrm{p}=0.006$ for gender effect and $\mathrm{p}=0.294$ for eating pattern effect (shown in Fig. 1).CfPWV values were $8.8 \pm 1.0$ and $7.9 \pm 0.9 \mathrm{~m} / \mathrm{s}$ for the male omnivores and vegetarians compared to $6.5 \pm 1.1$ and $6.5 \pm 0.9 \mathrm{~m} / \mathrm{s}$ for the female omnivores and vegetarians, respectively.

Blood pressure did not differ significantly between the omnivores and vegetarians $(\mathrm{SBP}=119.8 \pm 13.2$ and

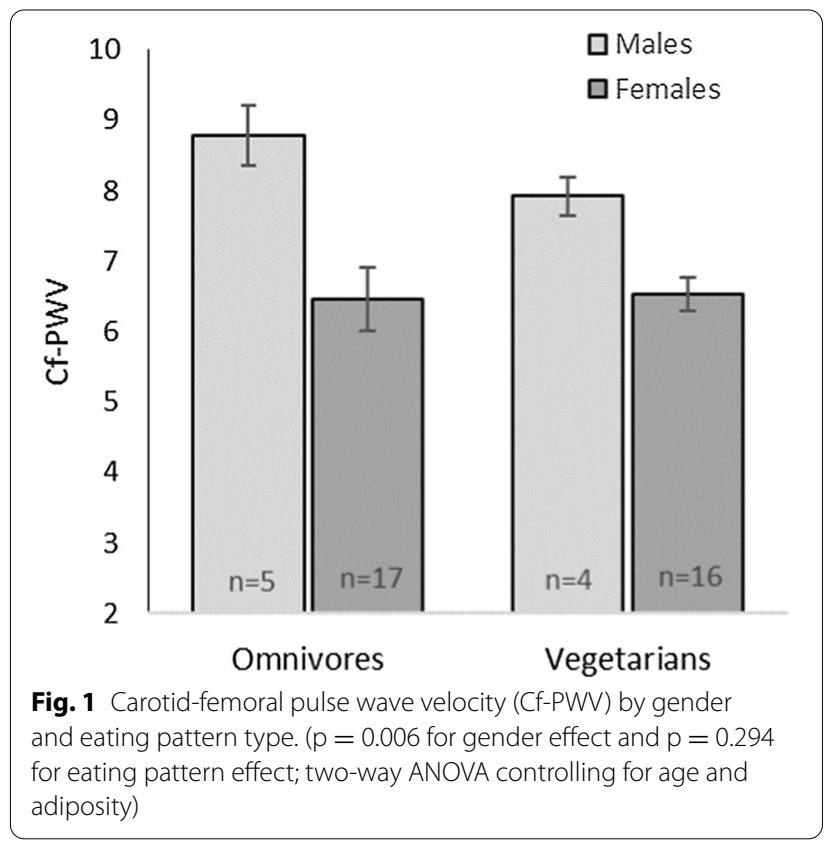

Table 2 Outcome variables by eating pattern type*

\begin{tabular}{lccl}
\hline & Omnivores & Vegetarians & p value \\
\hline Cf-PWV $(\mathrm{m} / \mathrm{s})$ & $7.0 \pm 1.5$ & $6.8 \pm 1.1$ & 0.073 \\
$\mathrm{SBP}(\mathrm{mmHg})$ & $119.8 \pm 13.2$ & $115.8 \pm 15.6$ & 0.891 \\
$\mathrm{DBP}(\mathrm{mmHg})$ & $73.9 \pm 8.2$ & $71.6 \pm 11.4$ & 0.998 \\
$\mathrm{CSBP}(\mathrm{mmHg})$ & $107.9 \pm 13.2$ & $103.5 \pm 15.2$ & 0.975 \\
CDBP $(\mathrm{mmHg})$ & $73.9 \pm 7.9$ & $72.3 \pm 11.5$ & 0.878 \\
Total cholesterol $(\mathrm{mg} / \mathrm{dL})$ & $203.4 \pm 39.7$ & $179.9 \pm 39.1$ & 0.119 \\
HDL cholesterol $(\mathrm{mg} / \mathrm{dL})$ & $63.8 \pm 18.5$ & $55.2 \pm 16.9$ & 0.013 \\
Total cholesterol/HDL ratio & $3.38 \pm 1.0$ & $3.46 \pm 1.0$ & 0.310 \\
LDL cholesterol (mg/dL) & $122.2 \pm 38.6$ & $105.1 \pm 32.1$ & 0.255 \\
Triglycerides $(\mathrm{mg} / \mathrm{dL})$ & $86.7 \pm 21.1$ & $97.9 \pm 41.8$ & 0.212 \\
Triglyceride/HDL ratio & $2.1 \pm 0.7$ & $1.6 \pm 0.9$ & 0.075 \\
hs-CRP $(\mathrm{mg} / \mathrm{L})$ & $1.5 \pm 2.2$ & $2.0 \pm 2.4$ & 0.459 \\
\hline
\end{tabular}

*Data are mean $\pm S D$; $p$-values represent univariate analysis controlling for age, adiposity, and gender 
$115.8 \pm 15.6 \mathrm{mmHg}$; $\mathrm{DBP}=73.9 \pm 8.2$ and $71.6 \pm 11.4 \mathrm{mmHg}$; CSBP $107.9 \pm 13.2$ and $103.5 \pm 15.2 \mathrm{mmHg}$; and CDBP $73.9 \pm 7.9$ and $72.3 \pm 11.5 \mathrm{mmHg}$ ). HDL cholesterol differed significantly between groups with omnivores displaying higher HDL concentrations than vegetarians; $63.8 \pm 18.5$ and $55.2 \pm 16.9 \mathrm{mg} / \mathrm{dL}$. Other lipid values $(\mathrm{p}>0.05)$, total cholesterol/HDL ratio $(3.38 \pm 1.0$ and $3.46 \pm 1.0$, for the omnivores and vegetarians, respectively; $\mathrm{p}=0.310$ ), and hs-CRP $(1.5 \pm 2.2$ and $2.0 \pm 2.4 \mathrm{mg} / \mathrm{L}$, respectively; $\mathrm{p}=0.541)$ did not differ between groups. Since the cardiometabolic factors were closely related, a multivariate ANOVA was conducted controlling for age, adiposity, and gender. There was no statistically significant difference between the diet groups on the combined dependent variables (LDL cholesterol, SBP, DBP, total cholesterol, triglycerides; $\mathrm{p}=0.269$ ).

\section{Discussion}

Cf-PWV is the gold-standard, non-invasive measure of arterial stiffness, and it is considered a reliable indicator of CVD risk [12, 13]. Although this study was not conducted to compare Cf-PWV by gender and eating pattern, and only $20 \%$ of the sample was male, these preliminary data suggest that a vegetarian eating pattern may be particularly advantageous for reducing CVD risk in young men. The data demonstrated a gender effect on Cf-PWV; however, the small portion of men in the sample did not permit adequate statistical power to demonstrate a significant gender by eating pattern effect.

Age is a strong predictor of arterial stiffness, and PWV increases $6-8 \%$ with each decade of life after age 50 [14]. The lower arterial stiffness for women versus age-matched men is noted mainly in the decades prior to, but not after, menopause [7]. Age ranged from 18 to 64 years in the current study (mean: $35.8 \pm 14$ years), with a similar age span by eating pattern by design. Hence, the gender effect noted herein may have been more pronounced in an exclusively young sample. Estrogen likely mediates this gender effect as many of its functions promote cardiovascular health, including the stimulation of nitric oxide, a potent vasodilator, the alleviation of oxidative stress, and the attenuation of salt sensitivity [15-18]. To our knowledge, the only other investigation comparing PWV in vegetarians and omnivores was a 2016 investigation that reported significantly lower PWV $(-8 \%)$ in healthy male vegetarians $(\mathrm{n}=44)$ versus a comparable omnivore control sample $(\mathrm{n}=44 ; 7.1 \pm 0.8$ and $7.7 \pm 0.9 \mathrm{~m} / \mathrm{s}$, respectively) [11]. These data suggest that in men, adoption of a vegetarian eating pattern may reduce CVD risk by improving PWV; however, this benefit may be limited in women, particularly those who are premenopausal. Future research examining PWV and CVD risk in younger versus older women who adhere to vegetarian eating as well as men across the lifespan would provide much-needed information on the benefits of meatless eating patterns to improve PWV and reduce CVD risk.

The difference in mean Cf-PWV between the two eating patterns was small $(0.2 \mathrm{~m} / \mathrm{s}$ or $3 \%)$; however, this difference is similar to reductions noted for PWV in controlled feeding trials. In a recent meta-analysis of 12 randomized controlled trials ( $\mathrm{n}=1007$ participants), $\mathrm{Chu}$ et al. observed $0.148 \mathrm{~m} / \mathrm{s}$ mean reduction in PWV in participants receiving fish oil supplements compared to controls $(\mathrm{p}=0.013)$ [19]. Trial durations averaged 14 weeks, and participant PWV at baseline averaged $\sim 8 \mathrm{~m} / \mathrm{s}$. A meta-analysis that examined the impact of soy isoflavone supplementation on PWV (four qualifying controlled, randomized trials encompassing 441 participants) demonstrated a significant reduction in average PWV $(-0.33 \mathrm{~m} / \mathrm{s} ; \mathrm{p}=0.02)$ [20]. Trial durations ranged from 4 to 12 weeks, and baseline PWV averaged $\sim 9 \mathrm{~m} / \mathrm{s}$. These investigations suggest that a $2-3 \%$ reduction in PWV is noteworthy. Moreover, a $0.2 \mathrm{~m} / \mathrm{s}$ reduction in PWV is meaningful considering the age-associated rise in PWV beginning at age $50: 0.43-0.93 \mathrm{~m} / \mathrm{s}$ per decade in normal and hypertensive populations, respectively [21].

\section{Limitations/conclusions}

This pilot study was limited by the small sample size and the relatively young age of the majority of female participants. Despite this, this is the first known examination of arterial stiffness in female vegetarians in comparison to matched omnivores, and the data can inform planning decisions for future observational studies and intervention trials. CVD will impact over one-third of Americans as they age. Diet-based strategies that effectively reduce CVD risk in men and women throughout the life cycle are warranted. It will also be prudent to address how smoking status might influence the progression of arterial stiffness in vegetarian populations.

\section{Abbreviations}

PWV: Pulse wave velocity; Cf-PWV: Carotid-femoral pulse wave velocity; SBP: Systolic blood pressure; DBP: Diastolic blood pressure; CSBP: Central systolic blood pressure; CDBP: Central diastolic blood; HDL: High density lipoprotein; LDL: Low density lipoprotein; hs-CRP: High-sensitivity C-reactive protein.

\section{Acknowledgements}

The authors thank Ginger Hook and Veronica Zamora for their excellent technical assistance.

\section{Authors' contributions}

STM: Study concept, data collection, manuscript draft and editing. CSJ: Study concept, statistical analyses, manuscript draft and editing. All authors read and approved the final manuscript.

\section{Funding}

This study was supported by the Graduate Research Support Program at Arizona State University. 


\section{Availability of data and materials}

Requests for data described in the manuscript may be made to the corresponding author.

\section{Declarations}

\section{Ethics approval and consent to participate}

This study protocol was reviewed and approved by the Institutional Review Board at Arizona State University, STUDY00009426. Written informed consent was obtained from all participants involved in the study.

\section{Consent for publication}

Not applicable.

\section{Competing interests}

The authors declare no competing interests.

\section{Author details}

${ }^{1}$ Department of Nutrition and Health Science, Ball State University, 1613 W Riverside Ave, Muncie, IN 47303, USA. ${ }^{2}$ College of Health Solutions, Arizona State University, 550 N. 3rd Street, Phoenix, AZ 85004, USA.

Received: 13 November 2021 Accepted: 4 February 2022

Published online: 19 February 2022

\section{References}

1. World Health Organization. Cardiovascular diseases. WHO Web. https:// www.who.int/news-room/fact-sheets/detail/cardiovascular-diseases(cvds). 2021; Accessed 8 Aug 2021.

2. Kesse-Guyot E, Vergnaud AC, Fezeu L, Zureik M, Blacher J, Péneau S, Hercberg S, Galan P, Czernichow S. Associations between dietary patterns and arterial stiffness, carotid artery intima-media thickness and atherosclerosis. Eur J Prev Cardiol. 2010;17(6):718-24. https://doi.org/10.1097/ HJR.0b013e32833a197f.

3. Albu A, Tache S, Mavritsakis N, Potoră C. Physical exercise and arterial stiffness in elderly. Palestrica Third Millennium Civil Sport. 2017;18(2):210-4

4. Bonarjee W. Arterial stiffness: a prognostic marker in coronary heart disease. Available methods and clinical application. Front Cardiovasc Med. 2018;5:64. https://doi.org/10.3389/fcvm.2018.00064.

5. Nabeel PM, Kiran VR, Joseph J, Abhidev VV, Sivaprakasam M. Local pulse wave velocity: theory, methods, advancements, and clinical applications IEEE Rev Biomed Eng. 2019;29(13):74-112. https://doi.org/10.1109/RBME. 2019.2931587.

6. Zhong Q, Hu MJ, Cui YJ, Liang L, Zhou MM, Yang YW, Huang F. Carotidfemoral pulse wave velocity in the prediction of cardiovascular events and mortality: an updated systematic review and meta-analysis. Angiology. 2018;69(7):617-29. https://doi.org/10.1177/0003319717742544.

7. Ogola BO, Zimmerman MA, Clark GL, et al. New insights into arterial stiffening: does sex matter? Am J Physiol Heart Circ Physiol. 2018;315(5):H1073-87. https://doi.org/10.1152/ajpheart.00132.2018.

8. Vaitkevicius PV, Fleg JL, Engel JH, O'Connor FC, Wright JG, Lakatta LE, Yin FC, Lakatta EG. Effects of age and aerobic capacity on arterial stiffness in healthy adults. Circulation. 1993;88(4 Pt 1):1456-62. https://doi.org/10. 1161/01.cir.88.4.1456 (PMID: 8403292).

9. Amireault S, Godin G. The Godin-Shephard leisure-time physical activity questionnaire: validity evidence supporting its use for classifying healthy adults into active and insufficiently active categorie. Percept Mot Skills. 2015;120(2):604-22. https://doi.org/10.2466/03.27.PMS.120v19x7.

10. Tremblay AJ, Morrissette H, Gagné JM, Bergeron J, Gagné C, Couture P. Validation of the Friedewald formula for the determination of low-density lipoprotein cholesterol compared with $\beta$-quantification in a large population. Clin Biochem. 2004;37(9):785-90. https://doi.org/10.1016/j.clinb iochem.2004.03.008

11. Acosta-Navarro J, Antoniazzi L, Oki AM, Bonfim MC, Hong V, AcostaCardenas P, Strunz C, Brunoro E, Miname MH, Salgado Filho W, Bortolotto $L$ A. Reduced subclinical carotid vascular disease and arterial stiffness in vegetarian men: the CARVOS study. Int J Cardiol. 2017;1(230):562-6. https://doi.org/10.1016/j.ijcard.2016.12.058.
12. Van Bortel LM, Laurent $S$, Boutouyrie $P$, et al. Expert consensus document on the measurement of aortic stiffness in daily practice using carotidfemoral pulse wave velocity. J Hypertens. 2012;30(3):445-8. https://doi. org/10.1097/HJH.0b013e32834fa8b0.

13. Wilson J, Webb AJS. Systolic blood pressure and longitudinal progression of arterial stiffness: a quantitative meta-analysis. J Am Heart Assoc. 2020;9(17): e017804. https://doi.org/10.1161/JAHA.120.017804.

14. Reference Values for Arterial Stiffness' Collaboration. Determinants of pulse wave velocity in healthy people and in the presence of cardiovascular risk factors: "establishing normal and reference values." Eur Heart J. 2010;31(19):2338-50. https://doi.org/10.1093/eurheartj/ehq165.

15. Somani YB, Pawelczyk JA, De Souza MJ, Kris-Etherton PM, Proctor DN. Aging women and their endothelium: probing the relative role of estrogen on vasodilator function. Am J Physiol Heart Circ Physiol. 2019;317(2):H395-404. https://doi.org/10.1152/ajpheart.00430.2018.

16. Kim JM, Kim TH, Lee HH, Lee SH, Wang T. Postmenopausal hypertension and sodium sensitivity. J Menopausal Med. 2014;20(1):1-6. https://doi. org/10.6118/jmm.2014.20.1.1.

17. Connelly PJ, Casey H, Montezano AC, Touyz RM, Delles C. Sex steroids receptors, hypertension, and vascular ageing. J Hum Hypertens. 2021. https://doi.org/10.1038/s41371-021-00576-7.

18. Hage FG, Oparil S. Ovarian hormones and vascular disease. Curr Opin Cardiol. 2013;28(4):411-6. https://doi.org/10.1097/HCO.0b013e32836205e7.

19. Chu Z, Wei Y, Hao Y, Wang J, Huang F, Hou M. Clinical effectiveness of fish oil on arterial stiffness: a systematic review and meta-analysis of randomized controlled trials. Nutr Metab Cardiovasc Dis. 2021;31(5):1339-48. https://doi.org/10.1016/j.numecd.2020.12.033.

20. Man B, Cui C, Zhang X, Sugiyama D, Barinas-Mitchell E, Sekikawa A. The effect of soy isoflavones on arterial stiffness: a systematic review and meta-analysis of randomized controlled trials. Eur J Nutr. 2021;60(2):60314. https://doi.org/10.1007/s00394-020-02300-6.

21. Diaz A, Tringler M, Wray S, Ramirez AJ, Cabrera Fischer El. The effects of age on pulse wave velocity in untreated hypertension. J Clin Hypertension (Greenwich). 2018;20(2):258-65. https://doi.org/10.1111/jch.13167.

\section{Publisher's Note}

Springer Nature remains neutral with regard to jurisdictional claims in published maps and institutional affiliations.

Ready to submit your research? Choose BMC and benefit from:

- fast, convenient online submission

- thorough peer review by experienced researchers in your field

- rapid publication on acceptance

- support for research data, including large and complex data types

- gold Open Access which fosters wider collaboration and increased citations

- maximum visibility for your research: over $100 \mathrm{M}$ website views per year

At $\mathrm{BMC}$, research is always in progress.

Learn more biomedcentral.com/submissions 